\title{
SEA ICE MOTION TRACKING FROM NEAR REAL TIME SAR DATA ACQUIRED DURING ANTARCTIC CIRCUMNAVIGATION EXPEDITION
}

\author{
Anja Frost ${ }^{1}$, Stefan Wiehle ${ }^{1}$, Suman Singha ${ }^{1}$, Detmar Krause ${ }^{2}$ \\ ${ }^{1}$ Deutsches Zentrum für Luft- und Raumfahrt (DLR), Maritime Safety and Security Lab Bremen \\ ${ }^{2}$ Deutsches Zentrum für Luft- und Raumfahrt (DLR), National Ground Segment, Neustrelitz
}

\begin{abstract}
Synthetic Aperture Radar (SAR) satellites are able to observe small and large scale structures in sea ice - in any weather, through clouds and darkness. In order to assist ship navigation during polar campaigns, we acquired SAR images along the ship course and provided them to navigators on board in near real time, utilizing the operational data processing chain of DLR ground station Neustrelitz. These "exclusive" acquisitions already helped to optimize the routes. SAR data, however, contain more information that is not easily visible, e.g. information about the local sea ice drift. In this paper, we explore the capabilities of a new software processor that is intended to retrieve high resolution sea ice drift fields from pairs of colocated SAR images, combining TerraSAR-X and Radarsat-2 images. The processor is foreseen to be integrated into the operational data processing chain at DLR ground station network sites.
\end{abstract}

Index Terms - Synthetic Aperture Radar, radar tracking, sea ice, navigation, correlation

\section{INTRODUCTION}

Navigation in Antarctic waters is challenging. Drifted by wind and currents, the sea ice situation can change significantly within hours. When ice is driven together, well navigable open water leads can close. When the pressure process continues, sea ice floes are forced upward into a line called ridge, or piled haphazardly one over another, forming an uneven surface [1]. Such sea ice is hard to cross, even for icebreakers.

Synthetic Aperture Radar (SAR) satellites make different structures within the sea ice visible. Due to their active radar antenna, they provide image data of the oceans and frozen waters independent of weather condition, cloud coverage, or sun illumination. Since decades, national ice services utilize SAR images in order to generate ice maps, which in turn are used for ship routing in polar waters. Nevertheless, regarding the topicality and resolution of these maps, there is room for improvement.
In order to assist ship navigation during polar campaigns, we established an operational data processing chain at DLR ground segment Neustrelitz that allows to downlink and process SAR images of different satellite missions acquired along the ship course and provide navigators on board with images in near real time (NRT). The processing chain was used in several campaigns $[2,3]$ and it has been shown that the supply with "exclusive" upto-date SAR images help to optimize routes. But SAR data contain more information that is not visually apparent from a single acquisition, e.g. information about the local sea ice drift.

In this paper, we explore the capabilities of a new software processor that is intended to derive high resolution sea ice drift fields from pairs of co-located SAR images recorded by the satellite missions TerraSAR-X (TS-X) and Radarsat-2 (RS2). That is, the processor is able to interlink SAR acquisitions of different orbits, different missions, and different Bands. In consequence, spatially and temporally near coincident acquisitions over Antarctica are possible multiple times per day, which allows frequent updates about the ice situation.

In our experimental results, we show sea ice drift fields generated from an image pair acquired during the Antarctic Circumnavigation Expedition in 2017. The drift fields have a resolution of $300 \mathrm{~m} \times 300 \mathrm{~m}$ and $150 \mathrm{~m} \times 150 \mathrm{~m}$. The high resolution reveals small variations within the sea ice motion. We observed two sea ice sheets drifting in opposite directions while in the single SAR images, they look like one continuous ice sheet. Hence, high resolution sea ice drift fields give a more detailed look into the current ice situation, indicating borders of ice sheets.

\section{ANTARCTIC CIRCUMNAVIGATION EXPEDITION}

In-depth knowledge of the cryosphere is crucial, not only for its preservation, but for the whole planet. The poles are strongly affected by climate change. On the other hand, polar regions influence the climate from the poles to the equator through interaction processes between ice, ocean, and atmosphere. In order to gain interdisciplinary knowledge, the Antarctic Circumnavigation Expedition 
(Dec. 2016 to Mar. 2017) bought together scientists from different countries on board the Russian research vessel Akademik Treshnikov for a three month journey around the Antarctic continent, providing circumpolar measurements of many variables [4]. The German Aerospace Center (DLR) supported the campaign with SAR acquisitions downlinked and processed in near real time at DLR ground segment in Neustrelitz. SAR quicklooks and L2 products, namely operational significant wave height retrieval, were delivered automatically and within 20 minutes after downlink.

\section{SEA ICE MOTION TRACKING}

For sea ice motion tracking from pairs of SAR images, we apply a phase correlation technique that is based on a frequency-domain representation of the SAR data and allows combining SAR images of different incidence angle ranges, different orbits, different Bands, and different missions. Phase correlation was first applied in [5] for image pattern matching, and used for SAR sea ice motion tracking in $[6,7]$. We presented successful first tests on phase correlation combining TS-X ScanSAR and RS2 ScanSAR Wide acquisitions in [3]. In this paper, we explore the capabilities of the method in higher resolutions up to $150 \mathrm{~m}$, using TS-X Stripmap mode and RS2 Fine Quad Pol mode.

Figure 1 illustrates the flowchart of our algorithm. First, co-registration is performed. Afterwards, drift vectors are estimated iteratively for defined image regions (in the following called image patches) in a hierarchical motion estimation framework.

\subsection{Iterative drift vector estimation}

The first step after co-registration is the extraction of image patches of size $N \mathrm{x} N$ pixel from each of the two images: patch $g(x, y)$ in the first image, with $(x, y)$ denoting the upperleft corner coordinates, and patch $h(x+u, y+v)$ in the second image, where the patch is shifted by the drift vector $(u, v)$, which initially is $(0,0)$.

The Hann function [8] is then applied to both patches to prevent discontinuities at the patch boundaries when performing the following complex fast Fourier transform (FFT). From the Fourier-transformed images, the normalized image cross spectrum is computed and transformed back to spatial domain:

$$
P C(u, v)=\mathfrak{I}^{-1}\left(\frac{\mathfrak{I}(g) \cdot \mathfrak{I}^{*}(h)}{\left|\mathfrak{I}(g) \cdot \mathfrak{I}^{*}(h)\right|}\right)
$$

$P C(u, v)$ shows a peak at $\left(u^{\prime}, v^{\prime}\right)$ indicating the pattern shift. The drift vector is then updated with the new values $\left(u+u, v+v^{\prime}\right)$. The procedure is iteratively repeated with a new patch $h$ until a peak at $\left(u^{\prime}=0, v^{\prime}=0\right)$ indicates that the optimal pattern match is found.

To cover the entire image, the patch position $(x, y)$ is changed according to the desired resolution of the drift field and the above procedure is repeated for each location.

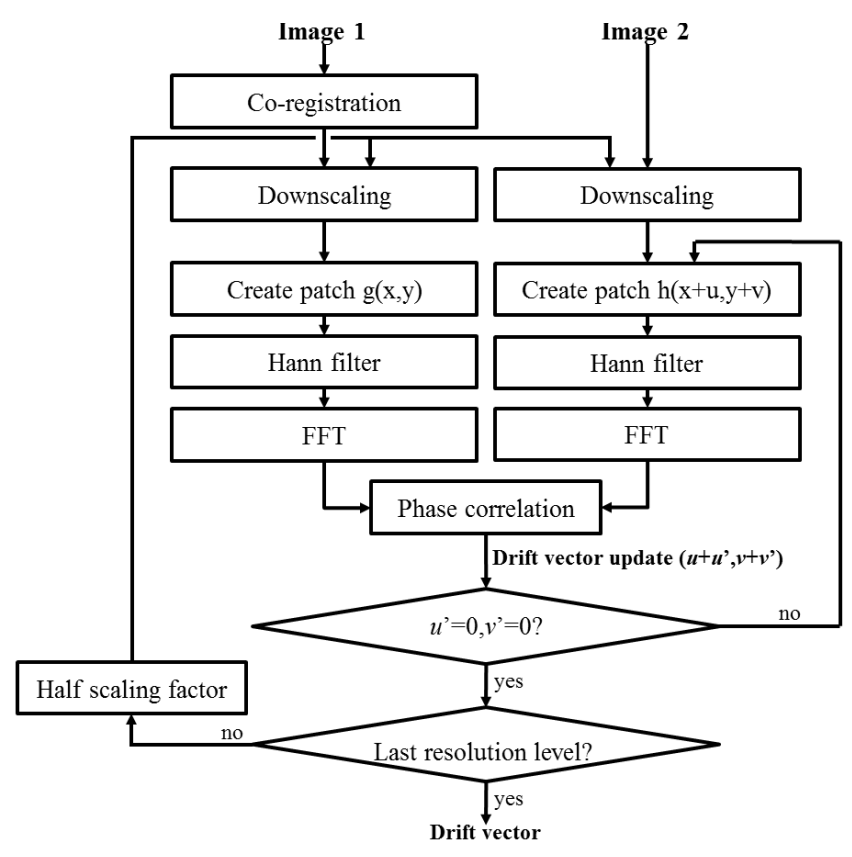

Figure 1: Flowchart for the estimation of one drift vector.

\subsection{Hierarchical processing}

For the hierarchical processing, the drift field calculation is repeated for each layer of a multi-resolution Gaussian image pyramid with a reduction factor of 2 . The size of the patches is kept constant at $N \times N$ pixels, so the coverage is high at the coarsest resolution and is halved with each higher resolution layer. This hierarchical approach makes a compromise between using large-scale image patches which may contain sea ice drifts in multiple directions resulting in ambiguous drift vector estimation, and small-scale image patches which cannot cover large motions of the sea ice.

\section{EXPERIMENTAL RESULTS AND DISCUSSION}

We analyze an image pair with a time interval of 27 minutes only, consisting of a TS-X Stripmap acquisition (2017/01/30 10:09 UTC) and a RS2 Fine Quad Pol acquisition (2017/01/30 10:36 UTC). Footprints are plotted in Figure 2.

Figure 4 shows the TS-X intensity image that is already co-registered to the following RS2 image (Figure 5). In the images, icebergs stand out bright as they give a relatively strong radar backscatter of about $-5 \mathrm{~dB}$ that is stronger than the backscatter from sea ice [9]. Areas of open water appear dark $(-20 \mathrm{~dB})$. Superimposed arrows in Figure 5 represent the sea ice drift field generated by our processor. In most parts, the sea ice drifted homogeneously westward. The drift speed in the northern part of the image was higher than in the southern part. In comparison, icebergs hardly move, but they show diverse movements. Iceberg (1) shifted slightly to Southwest. The faster sea ice drifted around it. Iceberg (2) rotated counter clockwise. Iceberg (3) moved to the East and, as the sea ice next to it moved into the opposite direc- 


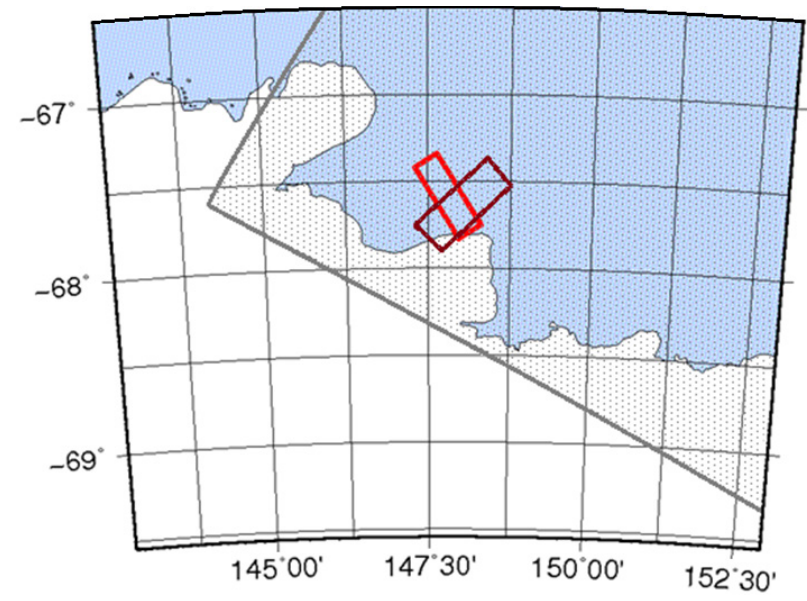

Figure 2: Footprint of a TS-X acquisition taken on 2017/01/30 10:09 UTC in ascending orbit with a center incidence angle of $43.34^{\circ}$ (light red) and of a RS2 acquisition taken on 2017/01/30 10:36 UTC in ascending orbit with a center incidence angle of $39.1^{\circ}$ (dark red). For deriving the motion field, we use HH channel only. For comparison, the footprint of the next Sentinel-1 acquisition taken approx. 30 hours later in extra wide swath mode is shaded in grey.

tion, it piled up a heap of ice floes. In the encircled zone, two ice sheets drift in opposite directions, but inspecting the individual SAR images, it appears like one continuous ice sheet. Hence, high resolution sea ice drift fields indicate hidden borders of sheering zones.

For a more detailed study, we repeated the drift vector estimation in a $150 \mathrm{~m}$ resolution and plotted the sea ice velocity (Figure 3). Small variations in the motion of the sea ice in the northern part become visible. Color jumps indicate the appearance of new open leads or compression zones.

In open water, there are no significant features to track and the drift retrieval fails.

\section{CONCLUSION}

We showed in our experimental results that a high resolution sea ice drift field, derived from pairs of sequential SAR images, reveals borders of individually moving ice sheets - more precisely ice sheet borders that are not visible in the single SAR image. In certain circumstances, the additional information on sea ice motion might help to improve ship navigation in ice infested waters.

The data were processed on a $2.7 \mathrm{GHz}$ single core CPU. The computing time for a $300 \mathrm{~m}$ resolution drift field amounts to 12 minutes only. The software processor is foreseen to be integrated into the operational data processing chain at DLR ground station network sites.

\section{ACKNOWLEGEMENT}

TS-X and RS2 data were acquired in context of EMS project. RS2 products (C) MDA.

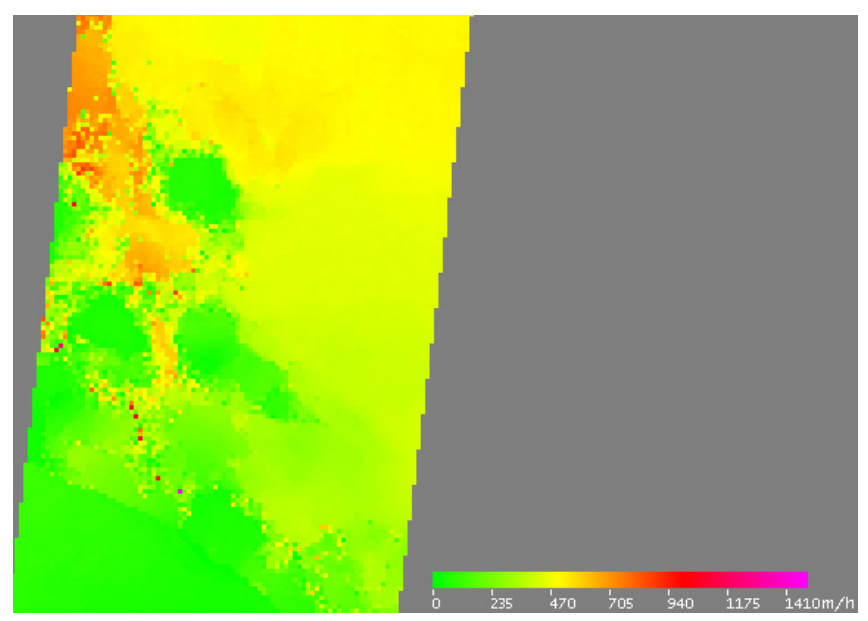

Figure 3: Sea ice velocity in $150 m \times 150$ m resolution.

\section{REFERENCES}

[1] Jackson, C. R., and Apel, J. R, Synthetic aperture radar: marine user's manual, US Department of Commerce, National Oceanic and Atmospheric Administration, National Environmental Satellite, Data, and Information Serve, Office of Research and Applications, 2004.

[2] S. Lehner, T. Krumpen, A. Frost, R. Ressel, T. Busche, and E. Schwarz, "First Tests on Near Real Time Ice Type Classification in Antarctica," Geoscience and Remote Sensing Symposium (IGARSS), IEEE International, pp. 4876-4879, 2014.

[3] A. Frost, S. Jacobsen, and S. Singha, "High Resolution Sea Ice Drift Estimation using Combined TerraSAR-X and RADARSAT-2 Data: First Tests," Geoscience and Remote Sensing Symposium (IGARSS), IEEE International, 2017.

[4] W. David, and D. Rod. "The Antarctic Circumnavigation Expedition - an interdisciplinary approach," Book of Abstracts, 2017.

[5] E. De Castro, and C. Morandi. "Registration of translated and rotated images using finite Fourier transforms." IEEE Transactions on Pattern Analysis \& Machine Intelligence, Vol. 5, pp. 700-703, 1987.

[6] J. Karvonen. Operational SAR-based sea ice drift monitoring over the Baltic Sea. In: Ocean Science 8.4: 473. 2012.

[7] T. Hollands. Motion tracking of sea ice with SAR satellite data. Diss. Universität Bremen, 2012.

[8] R. B. Blackman, J. W. Tukey, "Particular pairs of windows," The measurement of power spectra, from the point of view of communications engineering, Dover, New York, pp.95-101, 1959.

[9] A. Frost, R. Ressel, and S. Lehner. "Iceberg detection over northern latitudes using high resolution TerraSAR-X images," 36th Canadian Symposium of Remote Sensing-Abstracts, 2015. 


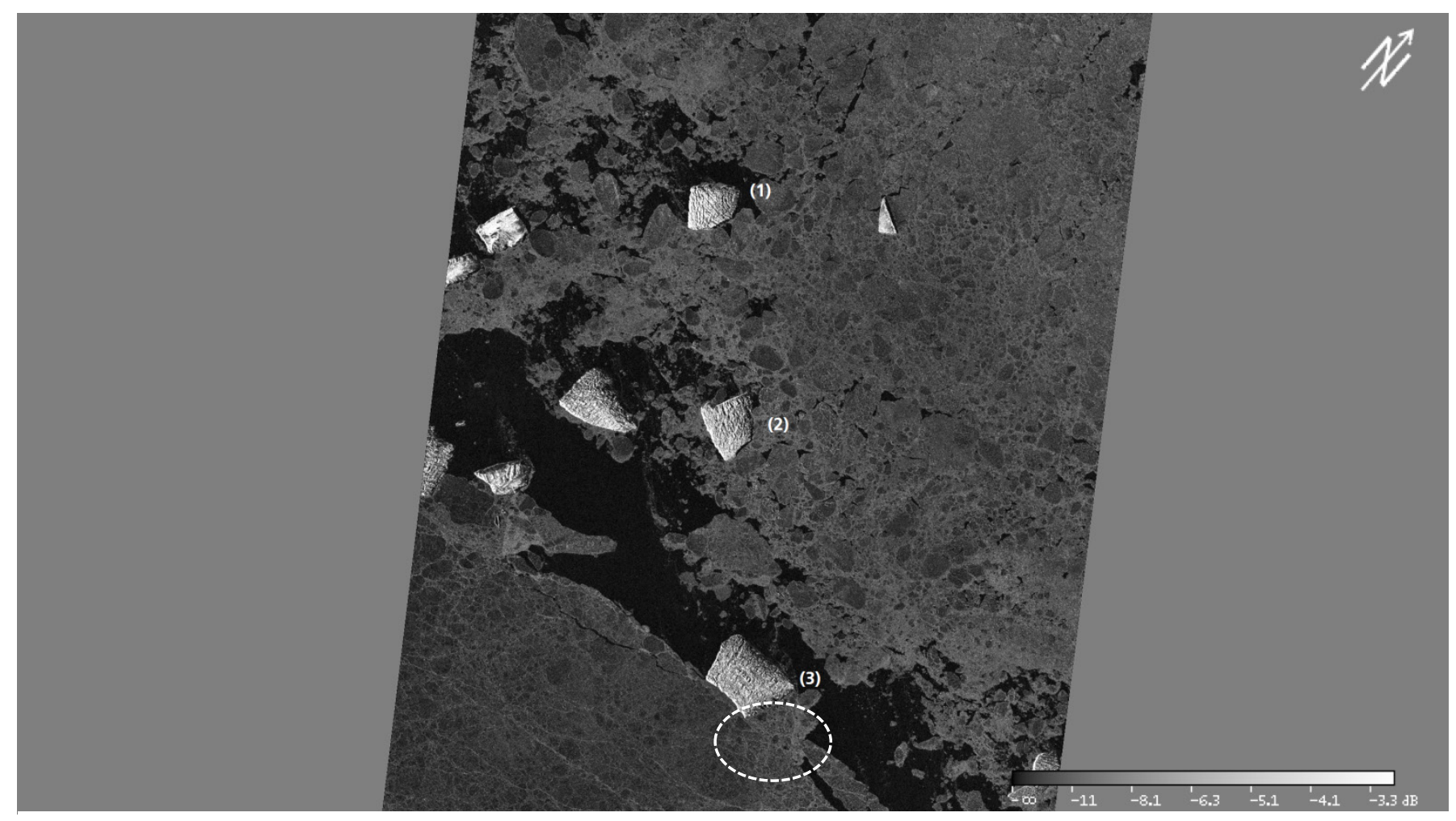

Figure 4: TS-X acquisition taken on 2017/01/30 10:09 UTC, calibrated and co-registered to the following RS2 acquisition.

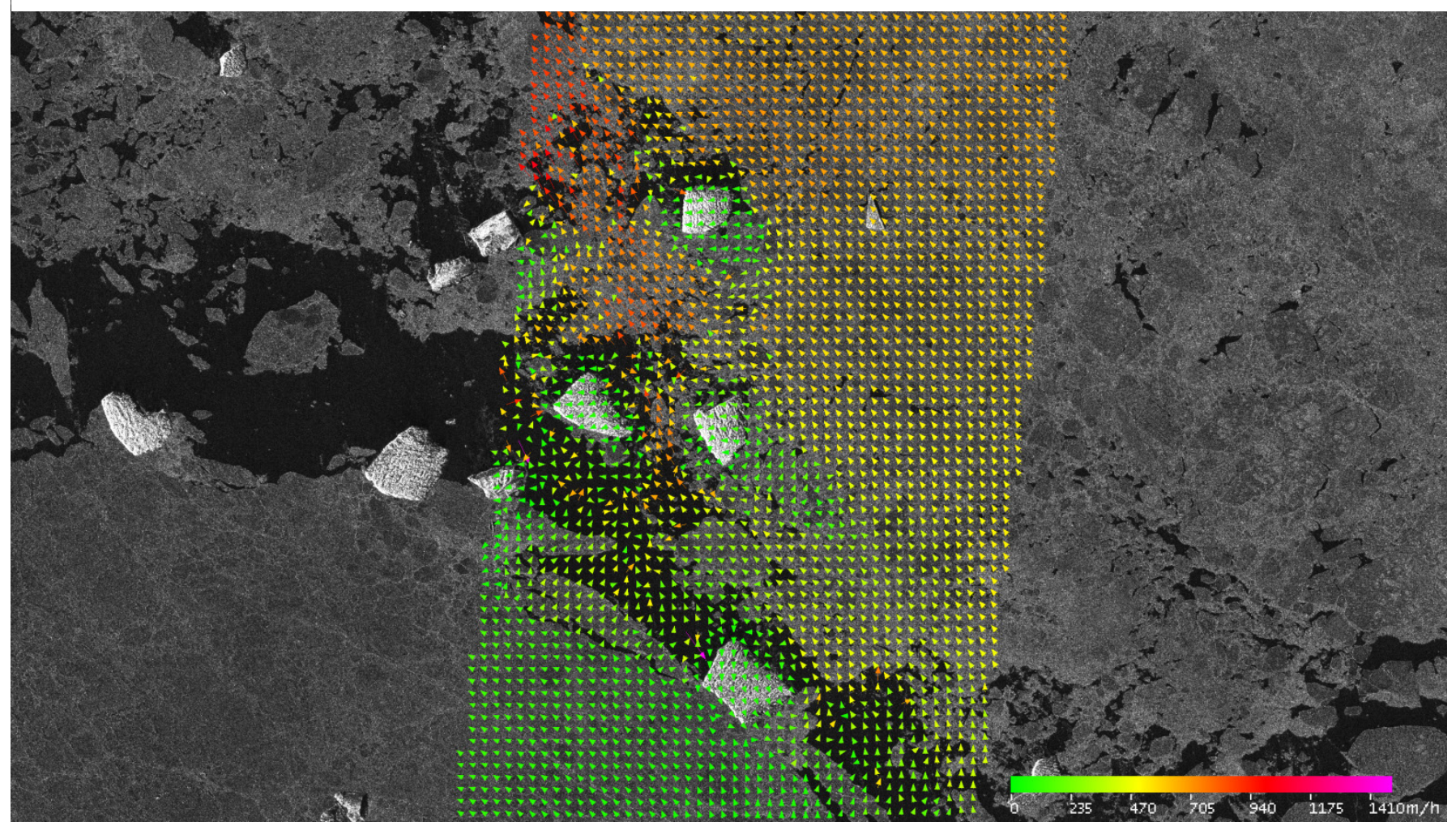

Figure 5: RS2 acquisition taken on 2017/01/30 10:36 UTC. Color-coded arrows show the sea ice drift field automatically generated from the RS2 image in the background and the TS-X image in Figure 4.The resolution of the drift field amounts to $300 \mathrm{~m} \times 300 \mathrm{~m}$. 